\title{
Emittance preservation during bunch compression with a magnetized beam
}

\author{
D. Stratakis
}

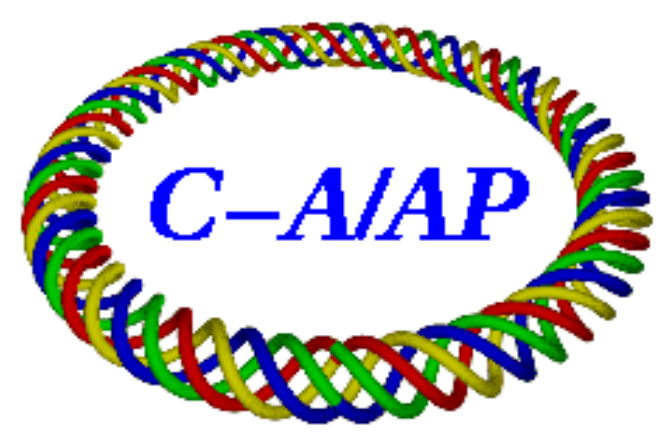

Collider-Accelerator Department Brookhaven National Laboratory Upton, NY 11973

\section{U.S. Department of Energy Office of Science, Office of Nuclear Physics}

Notice: This document has been authorized by employees of Brookhaven Science Associates, LLC under Contract No. DE-SC0012704 with the U.S. Department of Energy. The United States Government retains a nonexclusive, paid-up, irrevocable, world-wide license to publish or reproduce the published form of this document, or allow others to do so, for United States Government purposes. 


\title{
Emittance preservation during bunch compression with a
}

\section{magnetized beam}

\author{
Diktys Stratakis \\ Brookhaven National Laboratory, Upton NY 11973, USA
}

(Dated: September 02, 2015)

\begin{abstract}
The deleterious effects of coherent synchrotron radiation (CSR) on the phase-space and energy spread of high-energy beams in accelerator light sources can significantly constrain the machine design and performance. In this paper, we present a simple method to preserve the beam emittance by means of using magnetized beams that exhibit a large aspect ratio on their transverse dimensions. The concept is based on combining a finite solenoid field where the beam is generated together with a special optics adapter. Numerical simulations of this new type of beam source show that the induced phase-space density growth can be notably suppressed to less than $1 \%$ for any bunch charge. This work elucidates the key parameters that are needed for emittance preservation, such as the required field and aspect ratio for a given bunch charge.
\end{abstract}




\section{INTRODUCTION}

When electrons or other charged particles moving at relativistic speeds are forced by magnetic fields to follow curved trajectories they emit electromagnetic radiation in the direction of their motion, known as synchrotron radiation. Coherent synchrotron radiation (CSR) occurs when the emission of multiple electrons in a bunch is in phase, resulting in a quadratic dependence of the power emitted on the number of electrons participating. CSR is possible when either the entire electron bunch or any longitudinal structure of the bunch is comparable to the radiation wavelength $[1,2]$.

Electron beams with low transverse normalized emittance (sub- $\mu \mathrm{m}$ scale), short bunch length (at the sub-ps scale), and high peak current (up to thousands of amperes) is a key requirement [3, 4] in high-brightness light sources, plasma wave accelerations and linear colliders. However, such bunches cannot be produced directly, since forces from the mutual repulsion of electrons would destroy the brilliance of the beam within a short distance [5]. Instead, they are obtained by first accelerating a low peak current beam to relativistic energies and subsequently compressing the bunch length by several orders of magnitude to increase the peak current.

In most designs, the compression is achieved in magnet chicanes, where particles with different energies have different path lengths, so that a bunch with an energy distribution correlated with longitudinal particle position can shrink in length [6]. A successful compression system is one that compresses the bunch longitudinally while preserving the beam's transverse phase-space, referred to as the transverse beam emittance. In most cases, however, as a bunch propagates through the bends of a compressor, the tail-head interaction due to CSR can induce increased beam energy spread and transverse phase-space dilution that can severely degrade the 
downstream performance [7]. In addition, the CSR interaction is known to generate a microbunching instability in which large-amplitude current modulations can grow from smallamplitude, short-wavelength variations in the initial current profile.

Several techniques have been developed to mitigate the CSR effect [8-10]. For instance, utilizing asymmetry in a chicane design has shown success in partial nullification of emittance dilution when compared to the standard symmetric designs. Additionally, it has been shown that, in multi-stage compression systems, allocating more to the initial bunch compressors, while maintaining final compression, dampens the CSR effect in the final compressor where CSR is most detrimental. Though the techniques cited demonstrate effective reduction of the CSR induced emittance dilution, they tend to require many additional magnetic elements that can lead to further degradation of the longitudinal phase space and fall short of complete nullification.

In this paper, we present a simple and innovative solution to preserve the beam emittance during bunch compression. The method consists of generating a magnetized beam by immersing the photocathode in an axial magnetic field. After acceleration, the beam is transformed into a beam with asymmetric transverse emittances by using a set of three skew quadrupole magnets and by appropriate tuning of the lattice optics. With the aid of numerical simulations, we obtain a quantitative relationship between the required field and bunch charge. We show that emittance growth can be notably reduced to less than $1 \%$ with the proposed solution.

The outline of the paper is as follows: In Sec. II, we give an overview of the components of our accelerator beamline. Then, in Sec. III we detail a beam dynamics study for magnetized beams and present numerical results from modeling asymmetric particle beams on the aforementioned channel. Finally, we present our conclusions in Sec. IV. 


\section{ACCELERATOR BEAMLINE DETAILS}

Our simulations consider the photo-injector of the Brookhaven National Laboratory (BNL) Accelerator Test Facility (ATF) [11] which is shown schematically in Fig. 1. In brief, electron charges with variable charge $(\mathrm{Q} \leq 3.2 \mathrm{nC})$ are generated via photoemission from a metal photocathode located at the back plate of a 1.6 cell radiofrequency (RF) operating at $2856 \mathrm{MHz}$. The gun is surrounded by a solenoid magnet S1 which is used to correct for the space-charge induced correlated emittance growth. For magnetized beam production, the solenoid S2 is turned on to provide the desired magnetic field on the photocathode along with the proper focusing. The electron bunches exiting the rf gun are then accelerated by two three meter long $2.856 \mathrm{GHz}$ traveling wave structures, up to $58 \mathrm{MeV}$. Downstream of the second acceleration section, is a set of three skewed quadrupoles which as we will show shortly will be used to generate the required asymmetry between the two transverse planes to mitigate CSR. The beamline also incorporates a four-bend magnetic bunch compressor which, consists of four $44.7 \mathrm{~cm}$ rectangular dipoles with respective bending angles of $20^{\circ}$. The bending is assumed to be on the horizontal plane while the longitudinal dispersion is $\mathrm{R}_{56}=-9.3 \mathrm{~cm}$. During compressor operations, an energy-time correlation is imparted onto the electron bunch by operating the two travelling wave structures off-crest. The induced correlation allows for a change in the time of flight of the particles of the beam, effectively shortening the bunch length of the beam, thereby increasing the current. Detailed beam parameters as well as the required linac phase for compression is shown in Table I.

\section{BEAM DYNAMICS OF A MAGNITED BEAM}

The numerical simulations presented in this paper were carried out with ASTRA [12] and ELEGANT [13] codes. ASTRA is a macroparticle code based on a rotationally symmetric space- 
charge algorithm and incorporates a detailed model for the traveling wave structure. The laser was modeled by a radially uniform transverse distribution with $0.25 \mathrm{~mm}$ radius. In the beamline of Fig. 1, ASTRA was used to simulate the beam dynamics from its formation at the photocathode up to the entrance of the quadrupole triplet with 200k macroparticles. Then, these macroparticles were used as input in ELEGANT, a well-established code for CSR simulation, and tracked through the remaining lattice. The performance of the injector was thoroughly explored for a variety of charges ranging from $50 \mathrm{pC}$ to $2000 \mathrm{pC}$ and the simulation was terminated downstream of the bunch compressor at $\mathrm{z}=11.95 \mathrm{~m}$ from the photo-cathode. This point is denoted as OP in Fig. 1.

The evolution of the transverse emittance along the photo-injector line for a conventional nomagnetized ( $\mathrm{S} 2$ is off) $300 \mathrm{pC}$ round beam is shown in Figure 2(a). The plot clearly indicates that the strong CSR wakefields blow up the beam's emittance by about threefold. This occurs mostly within the third and fourth magnets where the beam is already compressed and the peak current is high. The growth in transverse emittance reflects the fact that CSR induces a variation of the particle energy that is correlated with longitudinal coordinate along the bunch. As a consequence, different bunch slices are deflected with different angles in a bending magnet, and this deflecting error distorts the transverse phase-space. Figure 2(b) illustrates this smearing effect, by showing the transverse phase-space at the chicane exit.

Our proposed cure for emittance growth is to immerse the photocathode in an axial magnetic field and thereby introduce an average canonical angular momentum $\langle L\rangle=e B_{0} \sigma_{c}^{2}$ where is $e$ the electron charge, $B_{0}$ is the axial magnetic field on the photocathode surface, and $\sigma_{c}$ is the rootmean-square (rms) transverse size of the drive-laser spot on the photocathode. As the beam exits the solenoidal field, the angular momentum is purely kinetic resulting in a beam coupled in the 
two transverse planes [14-16]. A set of three skew quadrupoles can apply the torque necessary to remove the angular momentum of the beam and to break the symmetry between the $\mathrm{x}$ and $\mathrm{y}$ plane and thus generate an asymmetric beam between the two transverse planes; see analysis in Refs. 16, 17. Following the procedure described in more detail in Ref. 17, the strengths of the quadrupoles are chosen such as to minimize the figure of merit $\mathrm{FOM}^{2}=\Sigma_{13}^{2}+\Sigma_{14}^{2}+\Sigma_{23}^{2}+\Sigma_{24}^{2}$ at the triplet exit, where $\Sigma_{i j}$ is the element of the covariance matrix $\Sigma$. The resulting quadrupole magnet settings were then used used in ELEGANT to track the beam throughout the skew triplet and the magnetic chicane.

In Figure 3 we progressively raise the beam's average canonical angular momentum at the cathode by means of increasing the peak field of coil S2. Then, when generate a flat beam by properly adjusting the triplet to remove the induced angular momentum and show results for two bunch charge cases: one with $Q=50$ pC [Fig. 3(a)] and one with $Q=1000$ pC [Fig. 3(b)]. Clearly, as $\langle L\rangle$ strengthens up the induced horizontal emittance growth at OP decreases. A salient feature of our study is that the horizontal emittance growth is suppressed to $<0.5 \%$ for beams with large transverse emittance ratios which is a direct result of the increased canonical momentum at the cathode. The optimum values for $\langle L>$ to achieve this are 9.6 and $60.8 \mathrm{neV} \mathrm{s}$ for $50 \mathrm{pC}$ and $1000 \mathrm{pC}$, respectively. Conversely, the vertical emittance remains unaffected during compression and close to its initial value at the chicane entrance. This suggests that a flat beam could be a promising solution for preserving low emittance in one plane during bending: a fact that could potentially benefit linear colliders [18]. Our simulation also captures well the dependence of the needed angular momentum for emittance preservation with bunch charge. Quantitatively, Fig. 3(b) shows that if the bunch intensity is increased by a factor of 20, the required $\langle L\rangle$ to push back to the original emittance is 6 times higher. As a result, the 
emittance growth can be fully controlled by the magnetic field and the value for full compensation is correlated to the bunch intensity. Evolutions of the transverse emittances for the two charges along the beamline of Fig. 1 for the optimum $\langle L\rangle$ are shown in Fig. 3 (right). Note that the dash line depicts the evolution if CSR is neglected. Note further that the emittance growth from CSR at OP is less than $0.5 \%$ in both cases. More details on the required beam parameters for emittance preservation are listed in Table I.

Figure 4 displays the particle distribution of a magnetized beam at OP. Since the $1000 \mathrm{pC}$ bunch charge requires a larger magnetic field for emittance preservation compared to the $50 \mathrm{pC}$ case, the generated asymmetry is also bigger resulting to a longer beam in the horizontal direction. Conversely to the results of Fig. 3, the smearing effect is absent and the phase-space remains linear by preserving its elliptical shape. The reason that a magnetized beam could mitigate the emittance growth and avoid phase-space distortion is as follows. The asymmetric beam maintains a larger bunch length at the entrance of the fourth dipole (where the CSR is expected to be the greatest) compared to the round beam and the minimum bunch length is only achieved at the end of the fourth dipole magnet, so the angular spread of the beam introduced by the CSR induced energy spread is insignificant during bending. Quantitatively, the bunch length of a round beam at the entrance of the fourth magnet is $264 \mu \mathrm{m}$ while the asymmetric beam is $1290 \mu \mathrm{m}$. At the end of the chicane, however, it gets compressed to $27.5 \mu \mathrm{m}$ which is $30 \%$ less compared to the equivalent round beam.

To go beyond and get a quantitative prediction over a wide range of beam intensities, we plot in Fig. 5 the simulated canonical angular momentum and corresponding emittance asymmetry for suppressing the horizontal emittance dilution to less than $0.5 \%$ as a function of the bunch charge. As expected, when the bunch charge increases, the required $\langle L\rangle$ of the beam at the 
cathode needs to increase, too. Assuming that the transverse rms beam size on the cathode remains fixed this also implies a larger field on the solenoid. Quantitatively, if the rms beam radius at the cathode is fixed at $0.8 \mathrm{~mm}$, the required field for a $50 \mathrm{pC}$ bunch is 150 Gauss while for a $2000 \mathrm{pC}$ bunch the required field is 1450 Gauss. The corresponding emittance asymmetry rises from 30 for the low-charge case to 80 for the high-charge case.

\section{SUMMARY}

In recent years, the demand for applications of electron beams with low transverse normalized emittance, short bunch length, and high peak current has increased dramatically. Most popular examples include high-brightness light sources, plasma wave accelerations and linear colliders. Unfortunately, space-charge at low-energy prevent the generation of short bunches $(1<\mathrm{ps})$ directly from the electron source and the commonly used process of magnetic bunch compression may unacceptably degrade the beam quality, due to significant emittance growth caused by CSR effects in the bends.

In this paper, we have presented a simple solution for preserving the beam emittance during bunch compression. The method relied on the generation of a magnetized beam by immersing the photocathode in an axial magnetic field. After acceleration, the beam was transformed into a beam with asymmetric transverse emittances by using a set of three skew quadrupole magnets and by appropriate tuning of the beamline optics. Our results suggest that such asymmetric beam has the potential to mitigate CSR in a magnetic chicane since it maintains a larger bunch length at the entrance of the fourth dipole of the compressor (where the CSR is expected to be the greatest) compared to an equivalent round beam. With the aid of numerical simulations, we have obtained a quantitative relationship between the required canonical angular momentum on the cathode and bunch charge to preserve emittance in both horizontal and vertical planes during 
compression. Finally, our simulations indicated that a flat beam could serve as a promising solution to generate ultra-short (sub-ps scale) bunches while maintaining a very low emittance (sub- $\mu \mathrm{m}$ scale) in one plane.

\section{ACKNOWLEDGEMENTS}

The authors are grateful to I. Ben-Zvi and Y. Jing for many useful discussions. This work supported by the U.S. Department of Energy, contract no. DE-AC02-98CH10886. 


\section{REFERENCES}

[1] B. E. Carlsten and T. O. Raubenheimer, Phys. Rev. E 51, 1453 (1995).

[2] Ya. S. Derbenev, J. Rossbach, E. L. Saldin, and V. D. Shiltsev, Deutsches ElektronenSynchrotron, Report No. TESLA-FEL 95-05, 1995.

[3] P. Emma et al., Nat. Photonics 4, 641 (2010).

[4] W. Ackermann et al., Nat. Photonics 1, 336 (2007).

[5] R.A. Kishek, S. Bernal, C. L. Bohn, D. Grote, I. Haber, H. Li, P. G. O'Shea, M. Reiser, and M. Walter, Physics of Plasmas 10, p. 2016 (2003).

[6] T. Ishikawa, H. Aoyagi, T. Asaka et al., Nat. Photonics 6, 540 (2012).

[7] Talman, Phys. Rev. Lett. 56, 1429 (1986).

[8] M. Borland, J. W. Lewellen, S. V. Milton, "A highly flexible bunch compressor for the APS LEUTLFEL", in proceedings of LINAC2000, Monterey,USA, August 2000.

[9] S. Di Mitri et al., Phys. Rev. Lett. 110, 014801 (2013).

[10] Y. Jing, Y. Hao, and V. N. Litvinenko, Phys. Rev. ST Accel. Beams 16, 060704 (2013).

[11] www.bnl.gov/atf

[12] K. Floetmann, ASTRA particle tracking code, http://tesla.desy.de/ meykopff

[13] M. Borland, Phys. Rev. ST Accel. Beams 4, 070701 (2001).

[14] R. Brinkmann, Y. Derbenev, and K. Flottmann, Phys. Rev. ST Accel Beams 4, 053501 (2001).

[15] K. -J. Kim, Phys. Rev. ST Accel. Beams 6, 104002 (2003).

[16] A. Burov, S. Nagaitsev, and Ya. Derbenev, Phys. Rev. E 66, 016503 (2002).

[17] P. Piot, Y. -E Sun, and K. -J. Kim, Phys. Rev. ST Accel. Beams 9, 031001 (2006).

[18] P. Chen and K. Yokoya, KEK Report No. 91-2, 1991. 
TABLES AND FIGURE CAPTIONS

Table I: Beam line simulation parameters

\begin{tabular}{lcccc}
\hline Parameter & Unit & Value & Value & Value \\
& & & & \\
\hline Bunch charge & $\mathrm{pC}$ & 50 & 500 & 1500 \\
Energy & $\mathrm{MeV}$ & 57.3 & 57.0 & 57.0 \\
Emittance, $\varepsilon_{\mathrm{n}, \mathrm{x}}$ & $\mu \mathrm{m}$ & 9.48 & 35.60 & 69.05 \\
Emittance, $\varepsilon_{\mathrm{n}, \mathrm{y}}$ & $\mu \mathrm{m}$ & 0.31 & 0.79 & 1.01 \\
Emittance ratio & & 31 & 45 & 68 \\
Laser pulse length (rms) & $\mathrm{ps}$ & 3 & 3 & 3 \\
Gun peak field & $\mathrm{MV} / \mathrm{m}$ & 110 & 110 & 110 \\
Gun phase & $\mathrm{deg}$. & -21 & -21 & -21 \\
Peak field of $\mathrm{S}_{1}$ & $\mathrm{~T}$ & 0.16 & 0.18 & 0.18 \\
Linac accelerating field & $\mathrm{MV} / \mathrm{m}$ & 12 & 12 & 12 \\
Linac phase & $\mathrm{deg}$. & -11 & -15 & -15 \\
\hline
\end{tabular}


FIG. 1 (Color online): Overview of our accelerator beamline illustrating the solenoidal lenses (S1 and S2), two linac sections, quadrupole triplet and the magnetic bunch compressor. Point OP depicts the end of the beamline.

FIG. 2 (Color online): (a) Evolution of e-beam in the bunch compressor using a single chicane. The blowup on the horizontal emittance is caused by the CSR effect; (b) Transverse phase-space at OP which is clearly showing a smearing effect induced by CSR. Note that if CSR is neglected the horizontal emittance remains flat (dashed line).

FIG. 3 (Color online): (a) Left: Induced emittance growth versus canonical angular momentum at $\mathrm{OP}$ for a $50 \mathrm{pC}$ beam. The emittance growth is nearly suppressed when $<L>=9.6 \mathrm{neV}$ s. Right: Emittance evolution along the beamline of Fig. 1 for $\langle L\rangle=9.6 \mathrm{neV}$ s with and without CSR effect included in the simulation model; (b) Left: Induced emittance growth at OP versus angular canonical momentum for a $1000 \mathrm{pC}$ beam. The emittance growth is almost suppressed when $\langle\mathrm{L}\rangle=60.8 \mathrm{neV}$ s. Right: Emittance evolution along the beamline of Fig. 1 for $<L>=60.8$ neV s with and without CSR effect included in the simulation model.

FIG. 4 (Color online): Beam distributions at OP: (a) Configuration space and horizontal phasespace for a $50 \mathrm{pC}$ beam; and (b) Configuration space and horizontal phase-space for a $1000 \mathrm{pC}$ beam.

FIG. 5 (Color online): Canonical angular momentum at the cathode (red) and required emittance asymmetry (blue) to fully suppress emittance growth at OP during magnetic bunch compression versus the bunch charge. 
Figure 1

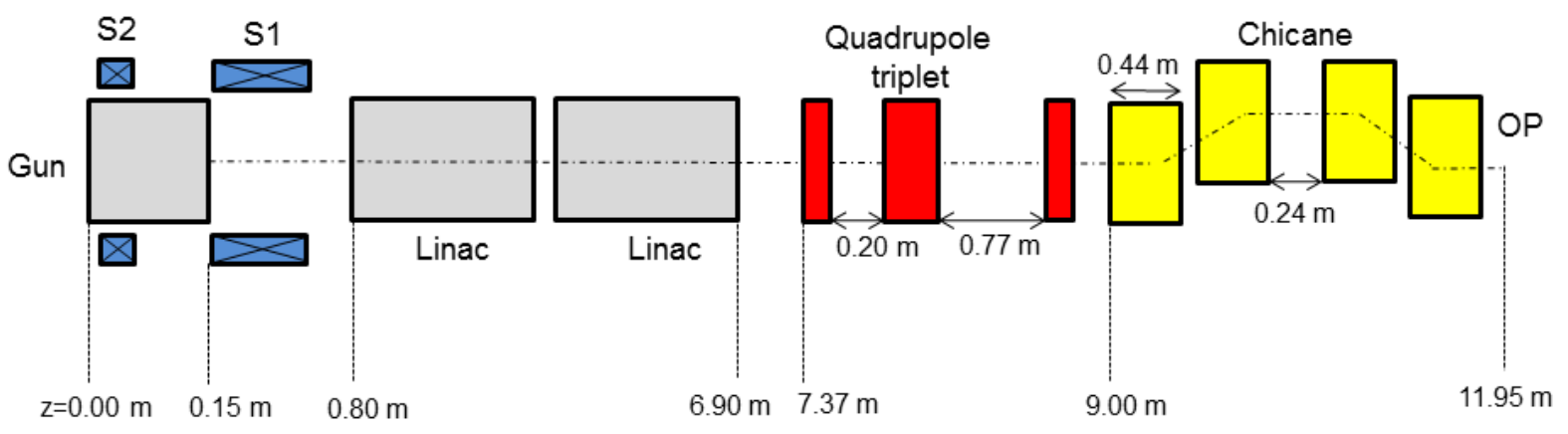


Figure 2

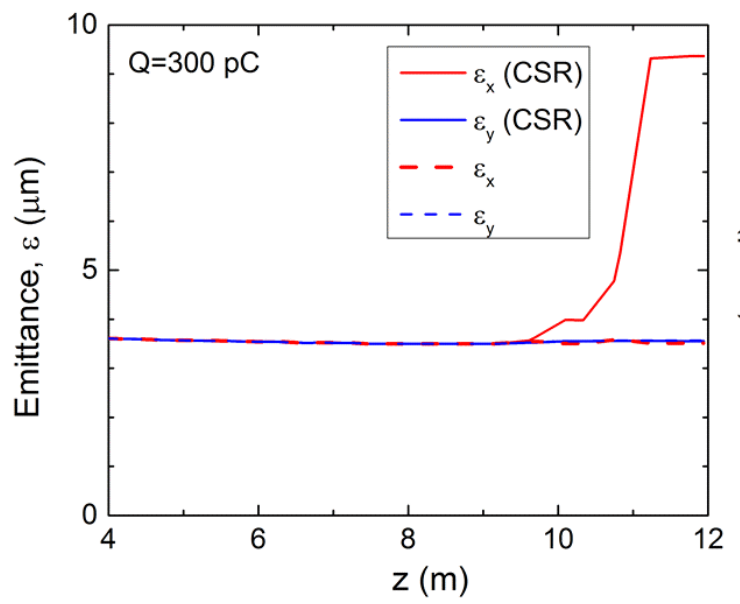

(a)

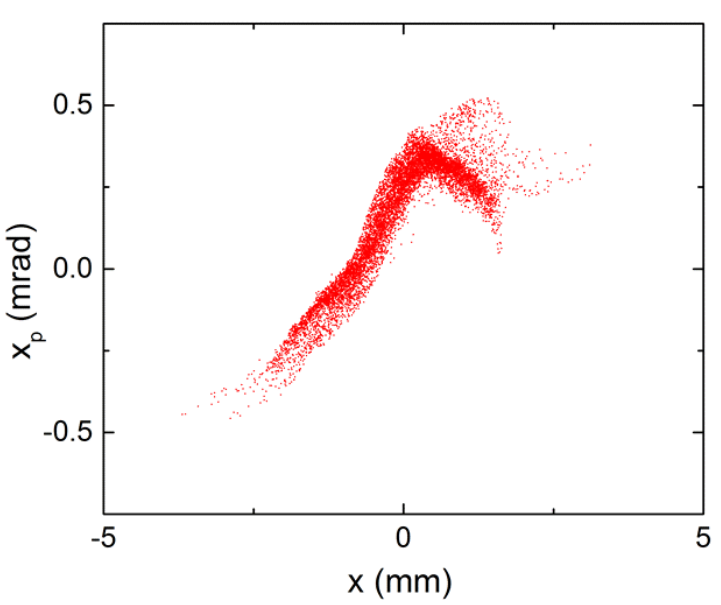

(b) 
Figure 3
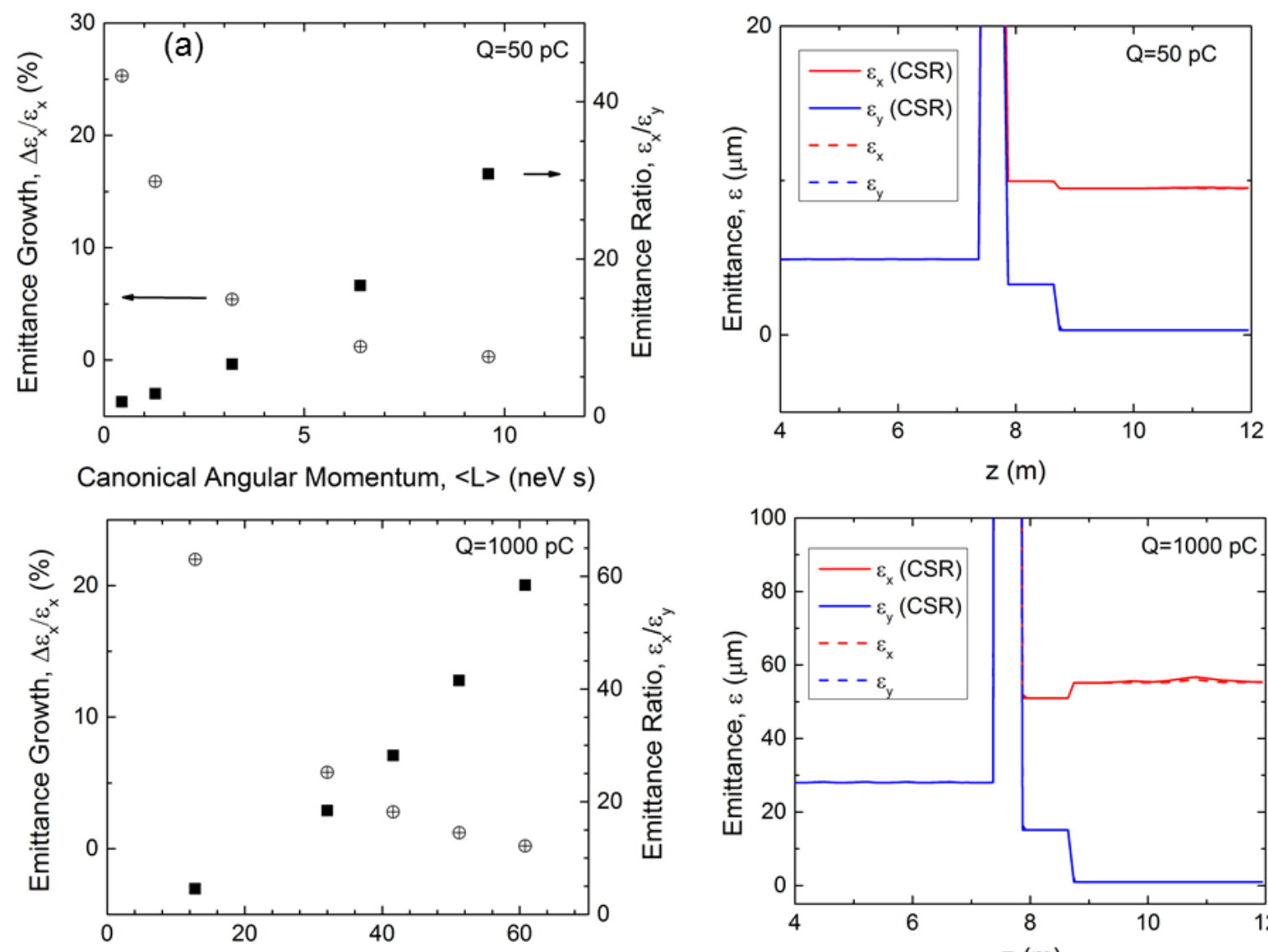

Canonical Angular Momentum, $<\mathrm{L}>$ (neV s)

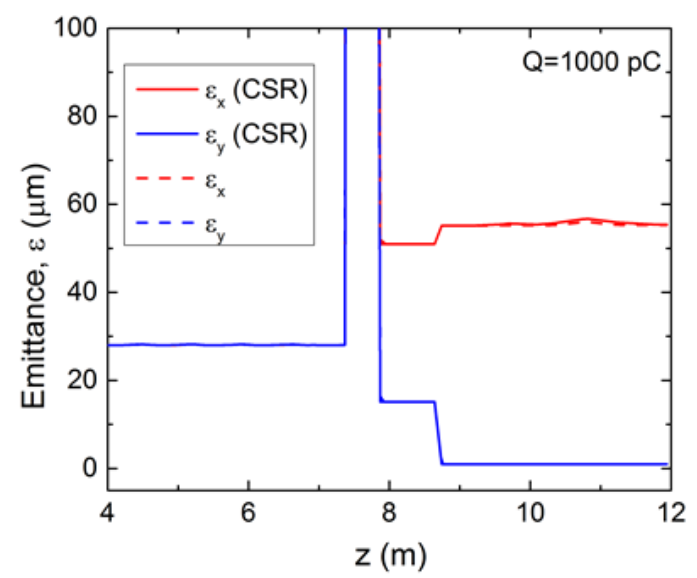


Figure 4

(a)

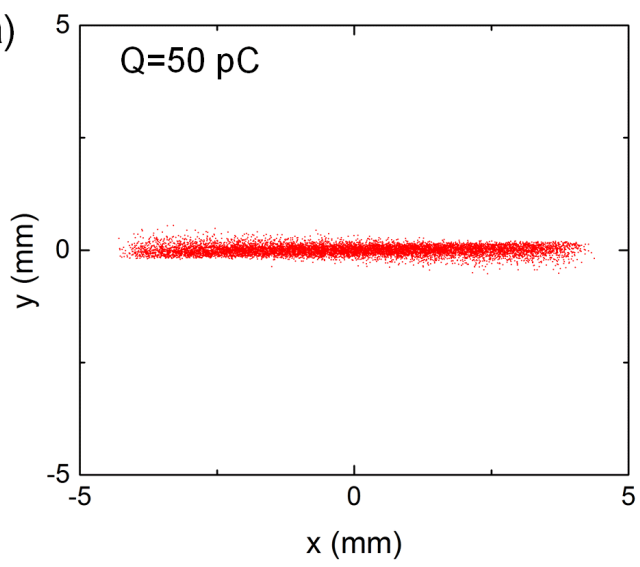

(b)

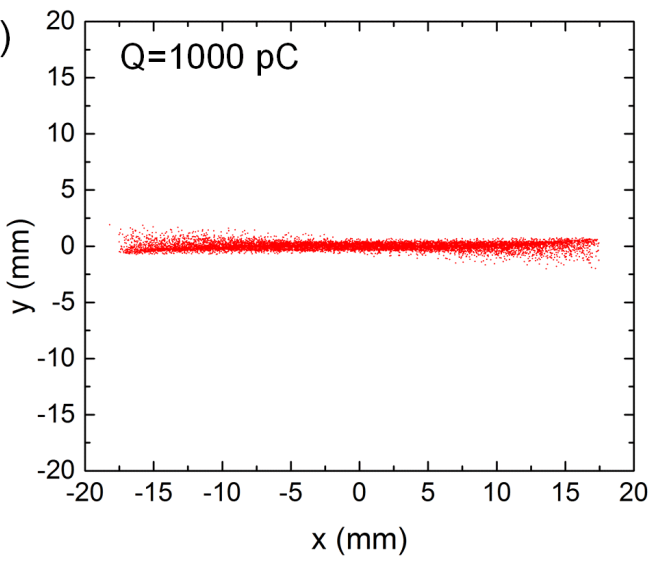

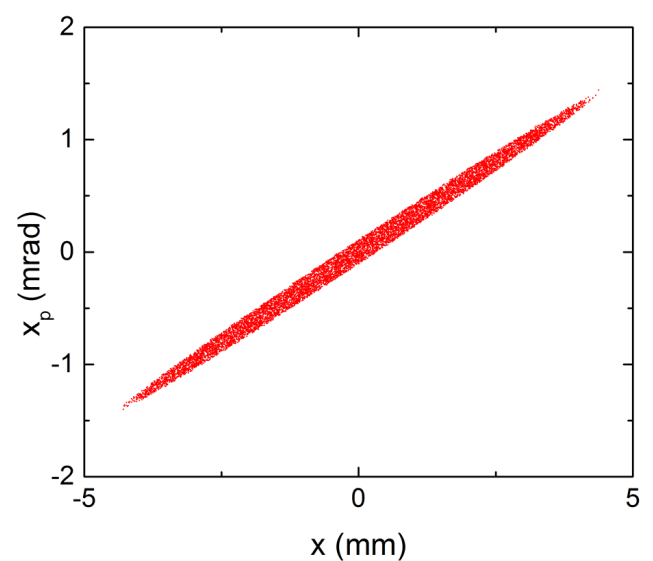

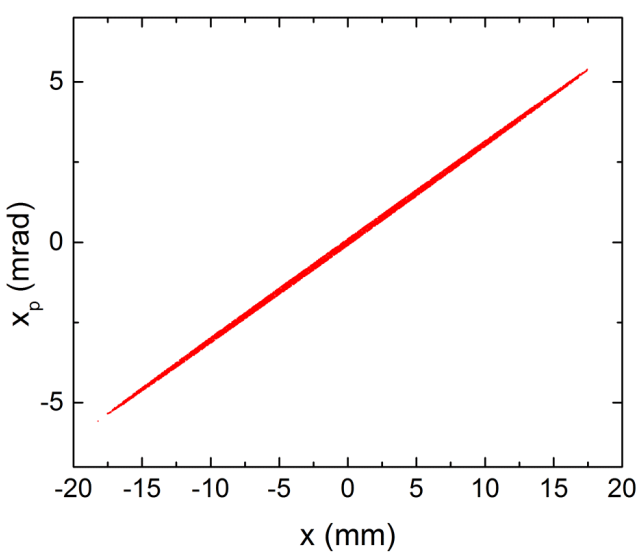


Figure 5

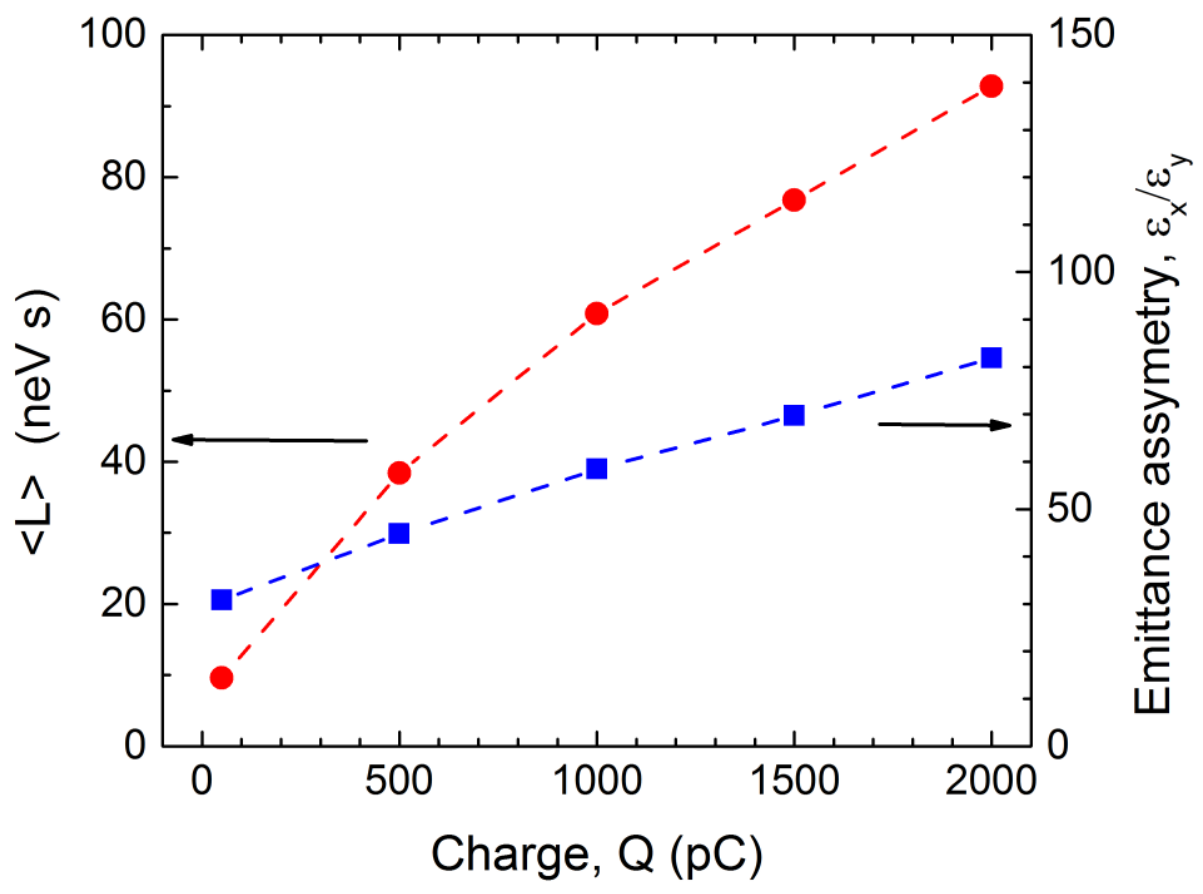

